\title{
Neutrophil airway influx by platelet-activating factor in asthma: role of adhesion molecules and $\mathrm{LTB}_{4}$ expression
}

\author{
J. Gabrijelcic*, A. Acuña*, M. Profita\#, A. Paternò\#, K.F. Chung ${ }^{\Uparrow}$, A.M. Vignola*, R. Rodríguez-Roisin*
}

Neutrophil airway influx by platelet-activating factor in asthma: role of adhesion molecules and $\mathrm{LTB}_{4}$ expression. J. Gabrijelcic, A. Acuña, M. Profita, A. Paternò, K.F. Chung, A.M. Vignola, R. Rodríguez-Roisin. (C) ERS Journals Ltd 2003.

ABSTRACT: Platelet-activating factor (PAF)-induced neutrophil lung sequestration may require cell surface adhesion molecules (macrophage-1 antigen (MAC-1) and lymphocyte function-associated antigen-1 (LFA-1)). In this randomised, double-blinded, crossover study, the neutrophil kinetics after PAF and Lyso-PAF (L-PAF) airway challenge were investigated in nine mild-intermittent asthmatics.

Neutrophils were measured in peripheral blood (PB) before and at 5, 15, 45 and $240 \mathrm{~min}$ after bronchoprovocation, and in induced sputum before and at $240 \mathrm{~min}$ after challenge. MAC-1 and LFA-1 expression were assessed by immunocytochemistry, and leukotriene $\mathrm{B}_{4}\left(\mathrm{LTB}_{4}\right)$ was measured by enzyme-immunoassay in induced-sputum supernatants.

Compared with baseline, neutrophils in PB decreased 5 min after PAF, while at 240 min neutrophils in induced sputum increased. Compared with baseline and L-PAF, PAF decreased the percentages of MAC-1- and LFA-1-positive neutrophils in PB at 5 min, but increased the percentages of MAC-1 and LFA-1 in neutrophil-induced sputum. Moreover, compared with baseline and L-PAF, PAF-induced sputum revealed higher $\mathrm{LTB}_{4}$ levels, a finding that correlated with the elevated number of neutrophils in induced sputum.

These findings suggest that macrophage-1 antigen and lymphocyte functionassociated antigen-1 are involved in platelet-activating factor-induced neutrophil lung traffic, and that this process is modulated by enhanced leukotriene $B_{4}$ release within the airways.

Eur Respir J 2003; 22: 290-297.
*Servei de Pneumologia i Al.lèrgia Respiratòria (ICPCT), Institut d'Investigacions Biomèdiques August Pi i Sunyer (IDIBAPS), Hospital Clínic, Universitat de Barcelona, Barcelona, Spain. " Istituto di Fisiopatologia Respiratoria, CNR, Palermo, Italy. "Dept of Thoracic Medicine, National Heart and Lung Institute, Imperial College, London, UK.

Correspondence: R. Rodríguez-Roisin, Servei de Pneumologia i Al.lèrgia Respiratòria, Hospital Clínic, Villarroel, 170, 08036-Barcelona, Spain.

Fax: 34932275404

E-mail: roisin@medicina.ub.es

Keywords: Inflammatory mediators, neutrophil kinetics, obstructive airway diseases

Received: October 252002

Accepted after revision: March 42003

This study was supported by the Comissionat per a Universitats i Recerca de la Generalitat de Catalunya (2001-SGR00386), a Research Grant (2000) from the European Respiratory Society (ERS), Ministerio de Sanidady Consumo (Redes Temátions de Investigación Cooperativa, $\mathrm{CO} 3 / 11)$ and a grant-in-aid from Esteve Group. A. Acuña was supported by Fundación Gran Mariscal de Ayacucho, Caracas, Venezuela, and Instituto de Cooperación Iberoamericana (ICI), Spain.
There is growing evidence that neutrophils may play an important role in different forms of asthma [1-6]. Indeed, increased numbers and different patterns of activation of neutrophils, and increased levels of neutrophil elastase and interleukin (IL)-8 have been shown previously [7, 8]. Abnormal expression of neutrophil and endothelial adhesion molecules has been shown in blood and sputum cells in mild atopic asthma [9] and during chronic obstructive pulmonary disease (COPD) exacerbations [10]. This may result from the increased sequestration of neutrophils in the pulmonary circulation during COPD exacerbations, leading to compartmentalisation of activated neutrophils within the lung [11]. However, the precise mechanism by which neutrophils traffic from pulmonary blood to the lung airspaces in both asthma and COPD remains, at least in part, undetermined. The release of leukotriene $\mathrm{B}_{4}\left(\mathrm{LTB}_{4}\right)$ may lead to neutrophil chemotaxis and neutrophil-endothelial interactions [12], and neutrophils are also an important source of $\mathrm{LTB}_{4}[13]$. $\mathrm{LTB}_{4}$ levels in induced sputum are elevated in COPD, correlate with elastase activity [14] and $\mathrm{LTB}_{4}$ is one of the possible mediators involved in neutrophil sequestration in asthma and COPD.

Platelet-activating factor (PAF) is an inflammatory mediator that may be involved in the pathogenesis of bronchial asthma.
It has been postulated that PAF-induced transient sequestration of neutrophils within the lung, which has been shown in normals [15-17] and asthmatics [18], could be a useful laboratory-induced model to investigate neutrophil kinetics in vivo and some of its mechanisms have been previously investigated by the current authors' group [15, 17, 18]. In the circulating blood compartment, PAF causes a transient neutropaenia followed by a neutrophilia, with recruitment of neutrophils in lung tissues and into the bronchoalveolar space $[16,17]$. There is also some evidence for activation of neutrophils, as the neutrophil volume was increased following PAF exposure [16]. Whether PAF induces the expression of integrins on the neutrophil, so as to increase its adhesion to the vascular endothelium or respiratory epithelium, to allow egress of the neutrophil from the vascular compartment into the tissues or from the latter into the bronchoalveolar space, respectively, remains elusive. PAF can also promote macrophage-1 antigen (MAC-1) (CD11b/CD18)-dependent neutrophil adhesion to endothelial cells through P-selectin $[19,20]$ and PAF by itself can increase leukocyte adhesion without altering vessel diameter [21]. Likewise, PAF can facilitate CD11b upregulation and L-selectin shedding induced by neutrophils [22], and can also cause neutrophil adhesion to the endothelium 
by upregulating intercellular adhesion molecule (ICAM)-1 [23]. Furthermore, PAF has the potential to promote neutrophil recruitment by stimulating the release of $\mathrm{LTB}_{4}$ by airway cells in asthmatic patients [24, 25], a mechanism also observed following MAC-1 overexpression by PAF [26].

Induced sputum is a useful technique to assess airway inflammation [27]. Using this tool, the present authors tested whether PAF induces neutrophil accumulation in the airways of patients with asthma through the modulation of neutrophil integrins. The effects of PAF or Lyso-PAF (L-PAF), its inactive metabolite [28], on the expression of $\beta_{2}$-integrins (MAC-1 and lymphocyte function-associated antigen-1 (LFA-1)) in neutrophils isolated from peripheral venous blood and induced sputum, and the levels of $\mathrm{LTB}_{4}$ in sputum supernatants, were therefore investigated in patients with mild asthma.

\section{Methods}

\section{Patients}

Nine nonsmoking patients with mild asthma were recruited for the study, which was approved by the ethical research committee, and gave informed written consent. The inclusion criteria were: age $>18$ yrs and $<45$ yrs; no respiratory infection or exacerbation of asthma within the preceding 6 weeks; forced expiratory volume in one second (FEV1) $\geqslant 70 \%$ predicted and $\geqslant 1.5 \mathrm{~L}$ after discontinuing bronchodilators for $12 \mathrm{~h}$, and positive provocative dose of methacholine causing a $20 \%$ fall in the FEV1 (PD20 $<1.9 \mu \mathrm{mol})$ on their first visit (see below); positive PAF response, as assessed by $\geqslant 35 \%$ increase in total respiratory system resistance $(R \mathrm{rs}) 5 \mathrm{~min}$ after PAF $(18 \mu \mathrm{g})$ inhalation; no previous treatment with oral steroids; and absence of any systemic or cardiopulmonary disease other than asthma. All subjects were atopic. Maintenance therapy included aerosol short-acting selective $\beta$-adrenergic agonists as needed (table 1).

\section{Study design}

Patients attended the laboratory on four separate occasions at 08:00 h. All visits were 1 week apart. On the first visit, the

Table 1.-Patient characteristics and baseline lung function before each challenge

\begin{tabular}{lc}
\hline Patient characteristics & \\
Patients n & 9 \\
Sex M:F & $5: 4$ \\
Age yrs & $24 \pm 1$ \\
FEV1 L & $3.63 \pm 0.17$ \\
FEV1 \% & $93 \pm 3$ \\
FEV $1 / \mathrm{FVC} \%$ & $78.5 \pm 2.9$ \\
Rrs $\mathrm{cmH} \mathrm{H}_{2} \mathrm{O} \cdot \mathrm{L}^{-1} \cdot \mathrm{s}^{-1}$ & $3.04 \pm 0.18$ \\
PD20 $\mu \mathrm{mol}$ & $0.51 \pm 0.08$ \\
PAF challenge & $86 \pm 12$ \\
Baseline lung function before each challenge & \\
FEV1 \% & \\
L-PAF & $92 \pm 5$ \\
PAF & $92 \pm 3$ \\
Rrs cmH $\mathrm{H}_{2} \mathrm{O} \cdot \mathrm{L}^{-1} \cdot \mathrm{s}^{-1}$ & \\
L-PAF & $3.59 \pm 0.26$ \\
PAF & $3.30 \pm 0.18$ \\
\end{tabular}

Data are presented as mean \pm SEM. M: Male; F: Female; FEV1: forced expiratory volume in one second; Rrs: respiratory system resistance; PD20: cumulative dose of methacholine that caused a $20 \%$ fall in FEV1; PAF: platelet-activating factor; L-PAF: Lyso-platelet-activating factor. \#: normal $>1.9 \mu \mathrm{mol}$; $\%$ \% increase in Rrs from baseline. clinical and functional assessment was performed. On the second visit, the patient attended for baseline sputum induction. On the next two visits, the patient attended for PAF or L-PAF bronchoprovocation, in a randomised, double-blinded, crossover manner. The PAF (C16, 1-O-hexadecyl-2-acetyl-snglycero-3-phosphocholine; $18 \mu \mathrm{g}$; Novabiochem AG, Laufelfingen, Switzerland) and L-PAF (C16, 1-O-hexadecyl-sn-glycero-3phosphocholine, fully saturated; $18 \mu \mathrm{g}$; Novabiochem AG) challenge was carried out as previously described [15]. A set of duplicate measurements was performed before the bronchoprovocation and at 5, 15, 45 and 240 min following PAF or L-PAF inhalation. These measurements consisted of $R$ rs (not at $240 \mathrm{~min}$ ), ventilatory and cardiac frequency recordings, and peripheral venous blood sampling. Sputum was also induced $240 \mathrm{~min}$ after completing the bronchoprovocation, based on a previous study [16]. All the challenges were clinically well tolerated by the participants who completed the study. As-needed short-acting $\beta_{2}$-agonists were only allowed until $24 \mathrm{~h}$ before the study.

\section{Measurements}

Rrs was measured by the forced oscillation technique [15] in each patient. A three-lead electrocardiogram, and cardiac frequency and arterial oxygen saturation via a pulseoximeter (HP M1166A; Hewlett-Packard, Boblingen, Germany), were continuously recorded throughout the study day. Peripheral blood (PB) samples were collected anaerobically through a catheter inserted into a peripheral vein.

\section{Separation of peripheral blood neutrophils}

Neutrophils and mononuclear cells were isolated using dextran sedimentation and centrifugation over Ficoll cushions, as previously described [29]. Subsequently, neutrophils and mononuclear cell counts and viability were assessed by a haemocytometer and trypan-blue dye exclusion, respectively, and were obtained from $2 \times 10^{5}$ cells fixed in acetone and stored at $-70^{\circ} \mathrm{C}$ for subsequent immunostaining [29].

\section{Induced sputum and processing}

Sputum was induced by the nebulisation of 3, 4 and $5 \%$ sterile hypertonic saline solution in sequence for $5 \mathrm{~min}$ from a De Vilbiss Ultraneb 99 ultrasonic nebuliser (Healthcare Inc., Somerset, PA, USA) through a mouthpiece without using valves or nose clips, as previously described [27, 29-31]. Fresh samples of induced sputum were processed immediately afterwards. Sputum plugs were selected, weighed, mixed with a four-times greater volume of $0.1 \%$ dithiothreitol (DTT) and processed, as previously described [4]. According to the weight of sputum plugs, sputum volume supernatants differed amongst samples. A minimal sample weight of $200 \mathrm{mg}$ was considered satisfactory for subsequent analysis. Supernatants were stored at $-70^{\circ} \mathrm{C}$, while cell pellets was resuspended in phosphate-buffered saline (PBS) to assess cell viability, total and differential cell counts, and for cytospins preparation. Differential cell counts were performed on cytocentrifuge preparations stained with May-Giemsa-Gruenwald. In all cases, 200 nonsquamous cells were counted by two blinded observers and results were expressed as percentage of total nonsquamous cells. 
Expression of adhesion molecules by peripheral blood and induced sputum neutrophils

Expression of CD11b/CD18 (MAC-1) and CD11a/CD18 (LFA-1) (DAKO A/S, Glostrup, Denmark) in both PB and induced sputum was evaluated in cytospins fixed in acetone using two specific monoclonal antibodies (mAbs): mAb 2LPM19c, reacting with the human 165-kd a-chain (CD11b) of the MAC-1 protein; and mAb E25.3, directed against the MHM24, reacting with the human 180-kd a-chain (CD11a) of the LFA-1 protein (DAKO A/S). These antibodies were used at dilutions of 1:100 for $1 \mathrm{~h}$, respectively. An irrelevant antibody anti-immunoglobulin $\mathrm{G}_{1}$ (DAKO) at a 1:10 dilution was used as a negative control. Immunoreactivity was revealed by the streptavidin-biotin alkaline-phosphatase technique. A total of 400 cells per slide were read independently by two observers. Results were expressed as percentage of the positive neutrophils minus total $\mathrm{PB}$ or induced sputum cells ratio. The interobserver coefficient of agreement was excellent (Kappa $=0.93$ ).

\section{Leukotriene $B_{4}$ measurement in induced sputum}

Sputum $\mathrm{LTB}_{4}$ levels were assessed by a specific enzymeimmunoassay method (Amersham International Plc, Little Chalfont, UK), as previously described [29]. The limit of detection for this method is $6 \mathrm{pg} \cdot \mathrm{mL}^{-1}$. The samples were diluted 1:5 before the assay and the value was corrected for the dilutions.

\section{Neutrophil chemotaxis assay}

Neutrophils obtained from PB of healthy donors were resuspended at a concentration of $1 \times 10^{6} \mathrm{~mL}^{-1}$ in PBS containing $\mathrm{CaCl}_{2}(0.5 \mathrm{mM})$ and $\mathrm{MgCl}_{2}(1 \mathrm{mM})$, and the supernatants from induced sputum of patients challenged with PAF and L-PAF were tested for their chemotactic activity on neutrophils. In addition, in order to evaluate whether chemotactic activity of induced sputum samples related to the presence of $\mathrm{LTB}_{4}$, experiments were performed in the presence and absence of an $\mathrm{LTB}_{4}$ receptor antagonist (LY223982, $10 \mu \mathrm{M}$; Eli Lilly, Basinstoke, UK). Chemotaxis was performed using a 48-well microchemotaxis chamber (Costar; Neuro Probe Inc., Cabin John, MD, USA), as previously described [29]. The volume of induced-sputum supernatant used in chemotactic assays was $27 \mathrm{~mL}$. Samples were not diluted before assaying for neutrophil migration. Migration was assessed by counting the number of cells that had migrated into the filter paper of the chemotaxis chamber with a pore size of $3 \mu \mathrm{m}$. Each experimental condition was performed in duplicate and three to four fields were assessed for cell migration. Chemotactic activity of the $\mathrm{LTB}_{4}$ standard was also evaluated. Furthermore, the number of cells migrating spontaneously in the presence of a solution $0.1 \%$ DTT, at the same concentration of induced-sputum supernatants (i.e. negative control), was subtracted from all measurements before data analysis.

\section{Statistical analysis}

Data are expressed as medians (25-75 percentiles). Multiple comparisons for $R$ rs and neutrophils and expression of adhesion molecules were determined using one-way analysis of variance (ANOVA) with Bonferroni's correction. Sputum cell differences were assessed using Wilcoxon's signed test with
Bonferroni's correction. Spearman's rank test was used for correlations. A $\mathrm{p}<0.05$ was considered significant.

\section{Results}

\section{Baseline findings}

There were no differences in baseline variables between each challenge day (tables 1 and 2), thereby indicating that the 1-week washout period after each challenge was sufficiently long enough to avoid any carry-over effects. Both ventilatory and cardiac variables (not shown) were within normal limits and remained unchanged throughout the whole study period.

Systemic, lung resistance and cellular changes after challenge

Eight patients noticed both facial flushing and breathlessness, two had cough, and one experienced mild rhinitis 5 min after PAF. By contrast, no symptoms were evident after L-PAF. At 5 min after PAF, compared with baseline, $R$ rs increased $(\mathrm{p}<0.0001)$ (table 2) while neutrophils in PB decreased $(p<0.004)$ (fig. 1). Subsequently, compared to $5 \mathrm{~min}$, neutrophils in PB increased at $15(\mathrm{p}<0.0005), 45(\mathrm{p}<0.0002)$ and $240 \mathrm{~min}(\mathrm{p}<0.0001)$. By contrast, no changes were seen after L-PAF.

Compared with baseline and L-PAF, both induced-sputum total cell count viability and the percentage of squamous cells after PAF were not significantly different (table 3). However, neutrophil percentages (neutrophils in induced sputum) increased $(\mathrm{p}<0.04$ and $<0.008)$ (fig. 2 ) whereas those of macrophages decreased $(p<0.02$ and $<0.008)$, respectively. By contrast, the percentages of eosinophils, lymphocytes and epithelial cells remained essentially unchanged after each challenge.

\section{Immunodetection of $\beta_{2}$-integrins in neutrophils from peripheral blood and induced sputum}

Compared with baseline and L-PAF, 5 min after PAF, the percentages of neutrophils in PB expressing MAC- $1(p<0.003$ each) and LFA-1 ( $<<0.004$ and $<0.02)$ decreased, respectively (table 2, and figs 1 and 2). A significant correlation was found between the reduction of the percentage of neutrophils in $\mathrm{PB}$ expressing MAC-1 and LFA-1 expression in PB and the decrease of neutrophils in $\mathrm{PB}$ ( $\mathrm{rho}=0.89$ and 0.87 , respectively; $\mathrm{p}<0.02$, each) $5 \mathrm{~min}$ after PAF. No changes were observed after L-PAF.

By contrast, compared with baseline (10.1 (5.5-12.1) \%) and L-PAF (10.2 (7.1-17.8) \%), the percentage of neutrophilinduced sputum expressing MAC-1 increased (to 25.0 (16.5$35.4) \%(p<0.02$ and $p<0.008$, respectively) after PAF exposure (figs. 2 and 3, and table 3). Similarly, compared with baseline (10.1 (4.4-12.5) \%) and L-PAF (10.3 (7.2-17.7) \%), the percentage of neutrophils in induced sputum expressing LFA1 increased (to $24.5(20.1-38.6) \%)(\mathrm{p}<0.008$ each) following PAF, although there were three patients who did not respond, indicating heterogeneity during PAF inhalation. A significant correlation was shown between the increased percentages of neutrophils in induced sputum expressing MAC-1 and LFA-1 and the elevated neutrophils in induced sputum (rho $=0.84, \mathrm{p}<0.02$; and $\mathrm{rho}=0.73, \mathrm{p}<0.04$, respectively) after PAF challenge. 


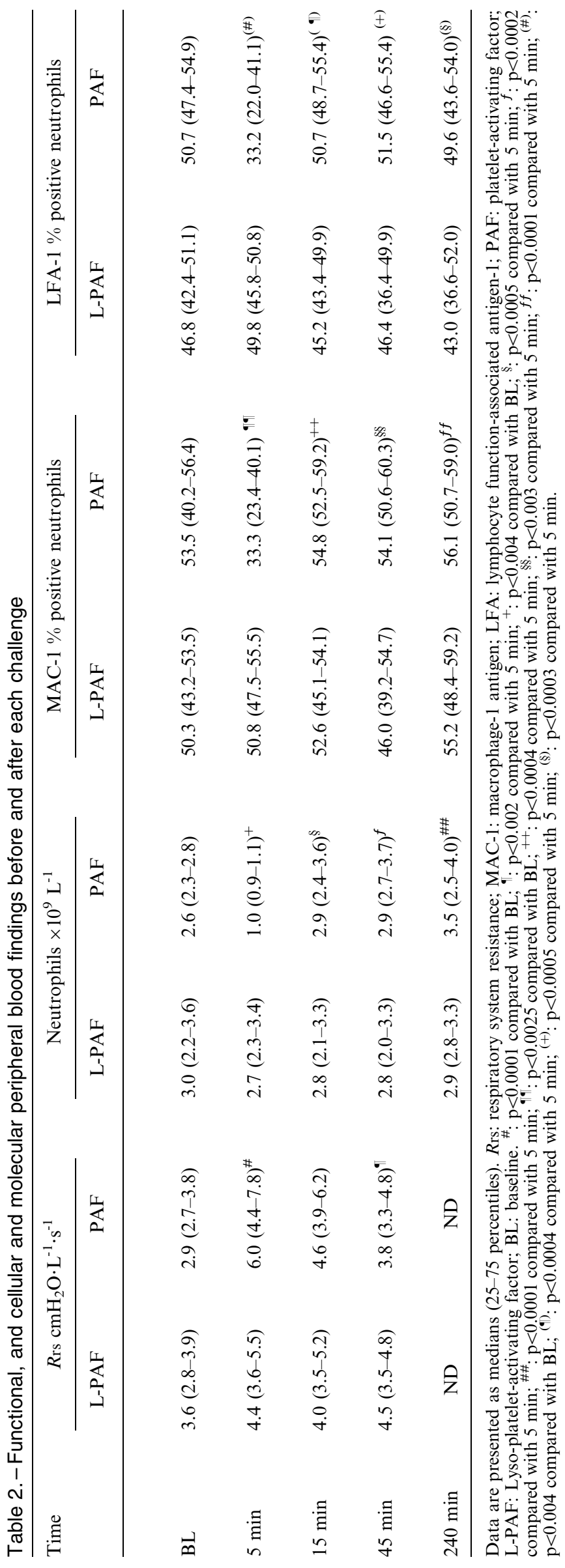

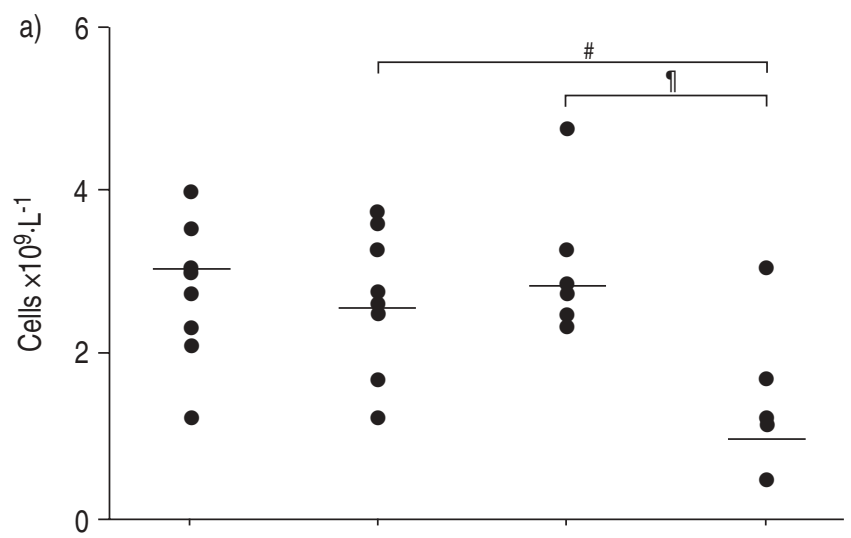
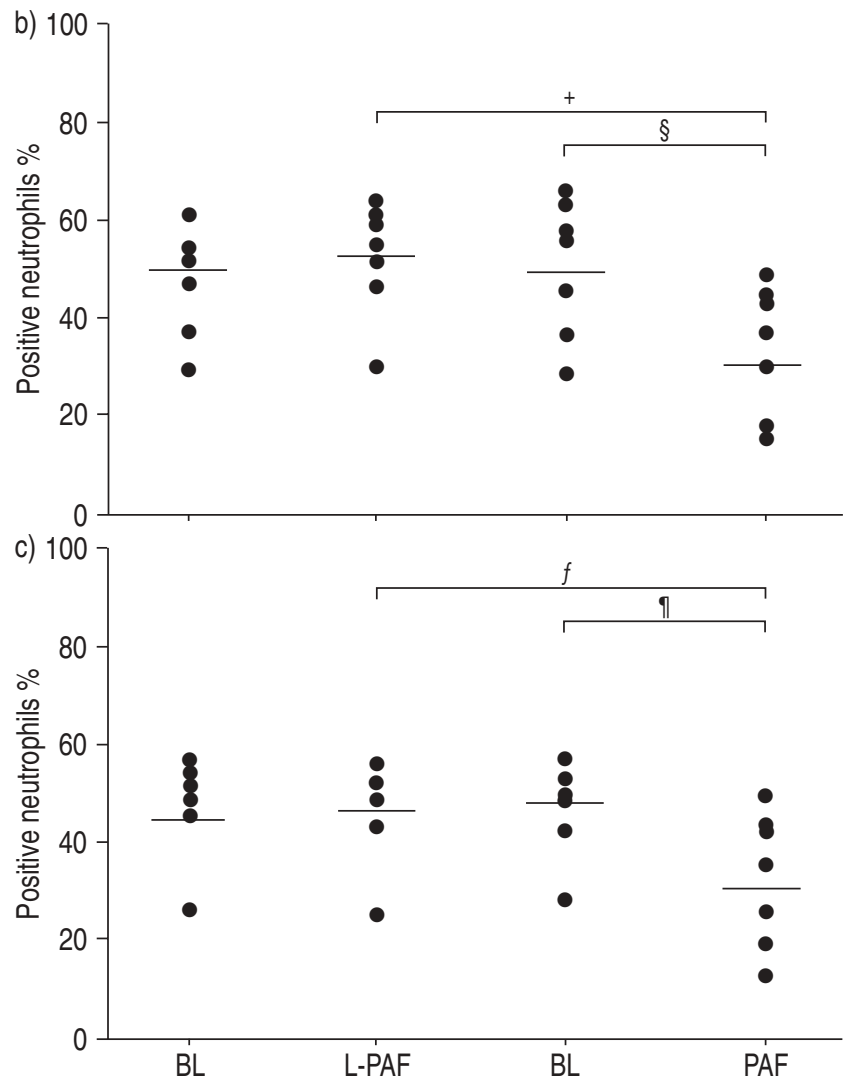

Fig. 1.- Peripheral blood a) neutrophils and their expression of b) macrophage-1 antigen (MAC-1) and c) lymphocyte function-associated antigen-1 (LFA-1) at their respective baselines (BL) and $5 \mathrm{~min}$ after Lyso-platelet activating factor (L-PAF) and PAF. Horizontal bars denote median values. ${ }^{\#}: \mathrm{p}<0.006 ;{ }^{\top}: \mathrm{p}<0.004 ;{ }^{+}: \mathrm{p}<0.003 ;{ }^{\S}: \mathrm{p}<0.002$; ${ }^{f}: \mathrm{p}<0.02$.

\section{Leukotriene $B_{4}$ measurement in induced-sputum supernatant}

Compared with baseline $\left(0.32(0.30-0.60) \mathrm{ng} \cdot \mathrm{mL}^{-1}\right)(\mathrm{p}<0.02)$ and L-PAF $\left(0.44(0.35-1.05) \mathrm{ng} \cdot \mathrm{mL}^{-1}\right)(\mathrm{p}<0.03), \mathrm{LTB}_{4}$ levels increased (to $1.36(1.09-2.73) \mathrm{ng} \cdot \mathrm{mL}^{-1}$ ) $240 \mathrm{~min}$ after PAF (fig. 4a), an increase that showed a close correlation with the elevated number of neutrophils in induced sputum ( $\mathrm{rho}=0.83$, $\mathrm{p}<0.02$ ) (fig. $4 \mathrm{~b}$ ).

\section{Neutrophil chemotaxis}

Compared with baseline (31.0 (25.7-40.7) cells $\cdot$ high-power field $^{-1}$ ) and L-PAF (46.0 (27.3-56.5) cells $\cdot$ high-power field ${ }^{-1}$ ), 
BL

$\begin{array}{cl}78.0 & (72.3-90.0) \\ 1.8 & (1.1-9.1) \\ 75.0 & (69.0-77.3) \\ 12.0 & (8.3-18.5) \\ 6.0 & (6.0-10.5) \\ 0 & (0-1.3) \\ 4.0 & (0.8-5.0) \\ 2.0 & (1.0-6.8) \\ 10.1 & (5.0-12.1) \\ 10.1 & (4.4-12.5)\end{array}$

L-PAF

PAF

Viability $\%$

Total cell counts $\times 10^{6} \cdot \mathrm{mL}^{-1}$

Macrophages \%

Neutrophils \%

Eosinophils \%

Lymphocytes \%

Epithelial cells \%

Squamous cells \%

MAC- $1 \%$

LFA-1 \%

$\begin{array}{cl}72.0 & (61.0-77.8) \\ 1.0 & (0.7-5.0) \\ 72.0 & (67.8-77.8) \\ 9.0 & (5.0-13.3) \\ 12.0 & (4.5-20.0) \\ 0 & (0-0.3) \\ 1.0 & (0-2.0) \\ 4.0 & (1.0-7.8) \\ 10.2 & (7.1-17.8) \\ 10.3 & (7.2-17.7)\end{array}$

$78.0(70.3-88.3)$

$1.8(0.3-3.5)$

$57.0(49.8-69.8)^{\#,}$

$28.0(16.5-36.8)^{+, \S}$

$12.0(4.8-14.8)$

$0(0-2.0)$

$0(0-4.3)$

$5.0(1.0-11.8)$

$25.0(16.5-35.4)^{\#,}$

$24.5(20.1-38.6)^{\S}$

Data are presented as median (25-75 percentiles). Macrophage-1 antigen (MAC-1) and lymphocyte function-associated antigen-1 (LFA-1) are expressed as \% positive neutrophils. BL: baseline; PAF: platelet-activating factor; L-PAF: Lyso-PAF. ${ }^{\#}$ : $\mathrm{p}<0.02$ compared with BL; ${ }^{\uparrow}$ : $\mathrm{p}<0.008$ compared with L-PAF; ${ }^{+}: \mathrm{p}<0.04$ compared with BL; ${ }^{\S}$ : $\mathrm{p}<0.008$ compared with BL and L-PAF.

there was an increase in neutrophil chemotactic activity (to $169.2(148.9-199.3)$ cells $\cdot$ high power $\cdot$ field $\left.^{-1}\right)(p<0.008$, each $)$ in induced sputum after PAF (fig. 5). Moreover, pre-incubation of neutrophils with the $\mathrm{LTB}_{4}$ receptor antagonist LY-223982 inhibited the chemotactic activity exerted by induced sputum after PAF by 52.8 (38.7-62.9) \%, as shown by a decreased number of migrating neutrophils (to 89.0 (68.0-99.5) cell$\mathrm{s} \cdot$ high power field $\left.{ }^{-1}\right)(\mathrm{p}<0.008)$ (fig. 5b).

\section{Discussion}

The most novel finding of the present study was an enhanced recruitment of neutrophils expressing MAC-1 and LFA-1 $4 \mathrm{~h}$ after PAF exposure within the airways of patients with mild asthma, and that such enhanced recruitment appeared to be driven by an increased production of $\mathrm{LTB}_{4}$, a potent neutrophil chemoattractant. It was also demonstrated that all these events were associated with a fall in blood neutrophils immediately after PAF and with an increased percentage of induced sputum neutrophils $4 \mathrm{~h}$ later.

These findings provide further evidence about some of the mechanisms underlying PAF-induced transient neutrophil pulmonary sequestration in mild asthma, an event observed in both healthy individuals [15-17] and asthmatics [18]. To the best of the current authors' knowledge, this is the first study in mild asthma showing that neutrophil recruitment into the airways following PAF exposure is the end result of the chemotactive effects elicited by increased levels of $\mathrm{LTB}_{4}$ on neutrophils expressing selective adhesion molecules, such as MAC-1 and LFA-1. The latter are critical for the regulation of neutrophil sequestration within the pulmonary capillaries and their subsequent transmigration across the endothelial pulmonary vascular barrier. Sequestration occurs in response to several intravascular inflammatory mediators and requires at least two sequential steps. First, rapid sequestration of neutrophils into the pulmonary circulation, which results in neutropaenia within $30 \mathrm{~s}$ [17], occurs throughout a process that does not require CD11/CD18 and is thought to involve a stimulus-induced decrease in the neutrophil's ability to deform [11]. Secondly, prolonged sequestration of these intracapillary neutrophils occurs for more than a few minutes, requiring CD11/CD18-mediated interactions with the endothelium [11]. Neutrophil migration throughout the pulmonary vessels is tightly regulated by adhesion molecules not only on neutrophils but also on endothelial cells such that, at sites of inflammation [32], ICAM-1 is highly expressed on activated endothelial cells. Thus, neutrophil adhesion may proceed via direct $\beta_{2}$-integrin ICAM-1 recognition [33]. In a recent in-vitro study [34], exposure to PAF was shown to increase neutrophil surface expression of MAC-1.

The results of the current study indicate that the increased
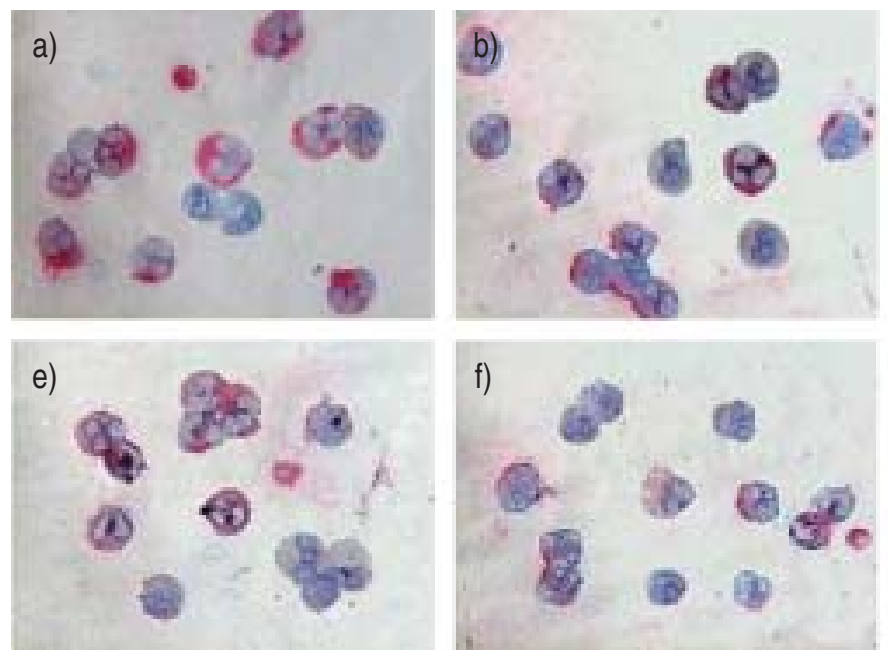
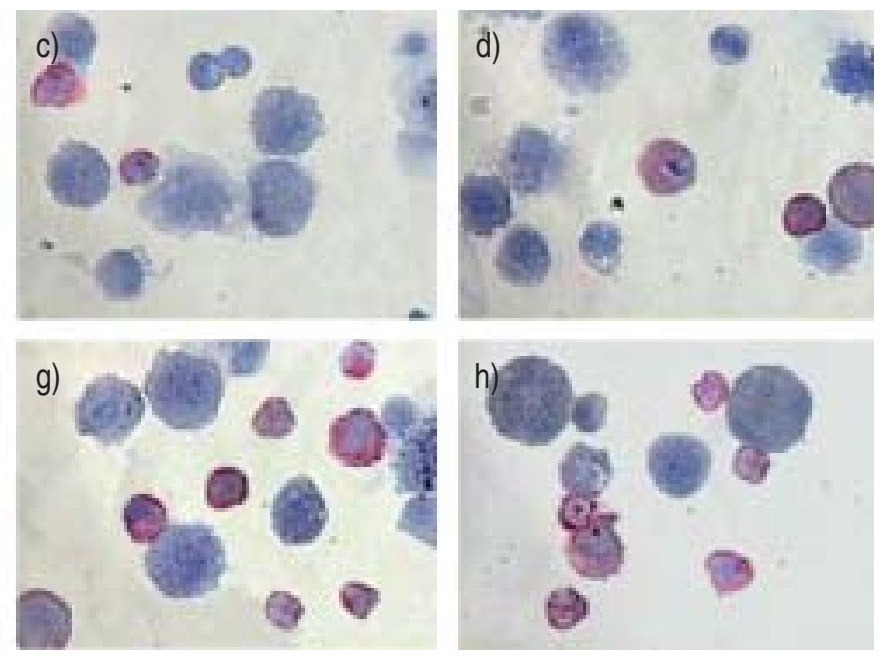

Fig. 2.-Representative immunostainings (in red) for macrophage-1 antigen (a, c, e and g) and lymphocyte function-associated antigen-1 (b, d, f and $\mathrm{h}$ ) by neutrophils isolated from peripheral venous blood (a, b, e and $\mathrm{f}$ ) and induced sputum (c, d, g and h) at baseline (top row) and at 5 (in peripheral blood) and $240 \mathrm{~min}$ (in sputum) after platelet-activating factor (PAF) challenge (bottom row). The data show decreased (peripheral blood) and increased (sputum) expression of both molecules after exposure to PAF compared with their corresponding basal staining. 

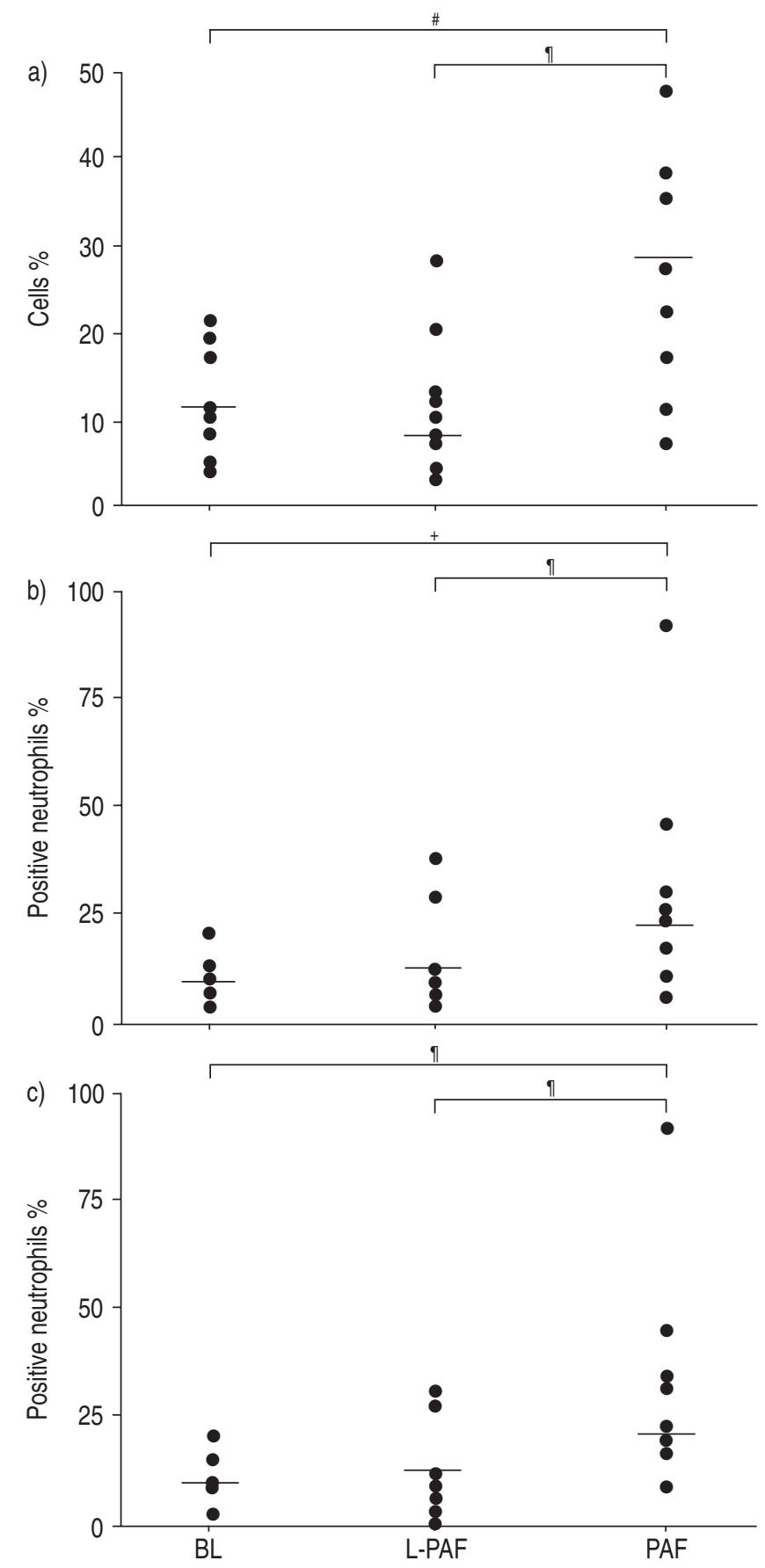

Fig. 3.-Percentages of induced sputum a) neutrophils and their expression of b) macrophage-1 antigen (MAC-1) and c) lymphocyte function-associated antigen-1 (LFA-1) at their respective baselines (BL) and $240 \mathrm{~min}$ after Lyso-platelet-activating factor (L-PAF) and PAF. Both MAC-1 and LFA-1 differences were still significant when their two highest individual values after PAF were excluded from the analysis $(\mathrm{n}=7 ; \mathrm{p}<0.032$ and $<0.02$, respectively). Horizontal bars denote median values. ${ }^{\#}: \mathrm{p}<0.04 ;{ }^{\dagger}: \mathrm{p}<0.008 ;{ }^{+}: \mathrm{p}<0.02$.

expression of recruitment of neutrophils through both MAC-1 and LFA-1 by PAF-induced sputum in asthmatics may play a critical role in the increased ability of white cells to adhere to and migrate across the bronchial and pulmonary vascular endothelium. This may allow for the rolling of neutrophils to stop at the intersection of endothelial cells while establishing rapid adhesion throughout LFA-1 [33, 35]. Subsequently, MAC-1 may promote sustained adhesion of neutrophils to
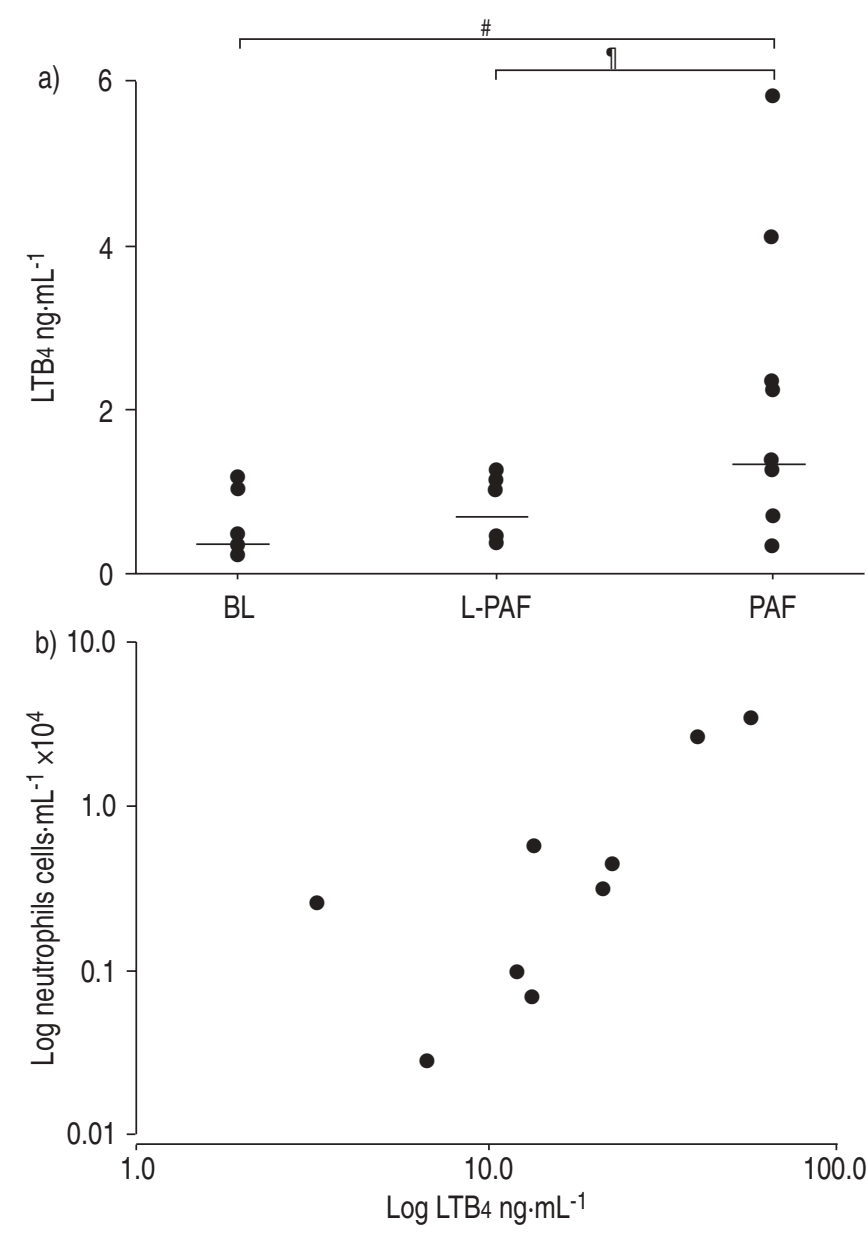

Fig. 4. - a) Individual leukotriene $\mathrm{B}_{4}\left(\mathrm{LTB}_{4}\right)$ levels in induced sputum supernatants at baseline (BL) and $240 \mathrm{~min}$ after Lyso-platelet-activating factor (L-PAF) and PAF. These differences were significant when the two highest $\mathrm{LTB}_{4}$ values after PAF were excluded $(\mathrm{n}=7 ; \mathrm{p}<0.05)$. Horizontal bars denote median values. ${ }^{\#}: p<0.02 ;$ : $p<0.03$. b) Plot between increased $\mathrm{LTB}_{4}$ levels in induced sputum supernatant and absolute neutrophils in peripheral venous blood $5 \mathrm{~min}$ after PAF challenge, both expressed on a logarithmic scale. rho: $0.83 ; \mathrm{p}<0.02$.

the endothelium, which is a prerequisite for their sequestration into the bronchial and pulmonary capillaries, and their subsequent transmigration into the pulmonary tissue. The fact that PAF challenge may play a key role in neutrophil recruitment is also suggested by the kinetics of the percentages of neutrophils expressing MAC-1 and LFA-1 expression in $\mathrm{PB}$ and sputum neutrophils. Indeed, compared with baseline and L-PAF, while the percentages of sputum neutrophils over total cells expressing MAC-1 and LFA-1 expressions were substantially increased after PAF, the percentage of peripheral venous blood neutrophils over total cells was decreased at a much earlier phase (i.e. $5 \mathrm{~min}$ ) after exposure. This data suggests, therefore, that MAC-1 and LFA-1 play a role in neutrophil recruitment after $\mathrm{PAF}$, and represent a prerequisite for neutrophil migration across the endothelial barrier. This is further supported by in-vitro evidence showing that PAF increased MAC-1 expression by neutrophils, which results in both integrin-dependent cell adhesion and extravasation [26, 34].

Nonetheless, expression of $\beta_{2}$-integrins per se, although important, does not seem to be the single mechanism required for an enhanced neutrophil accumulation in the lung. Indeed, leukocyte migration is a rather complex process in which the expression of adhesion molecules is only one of the steps 

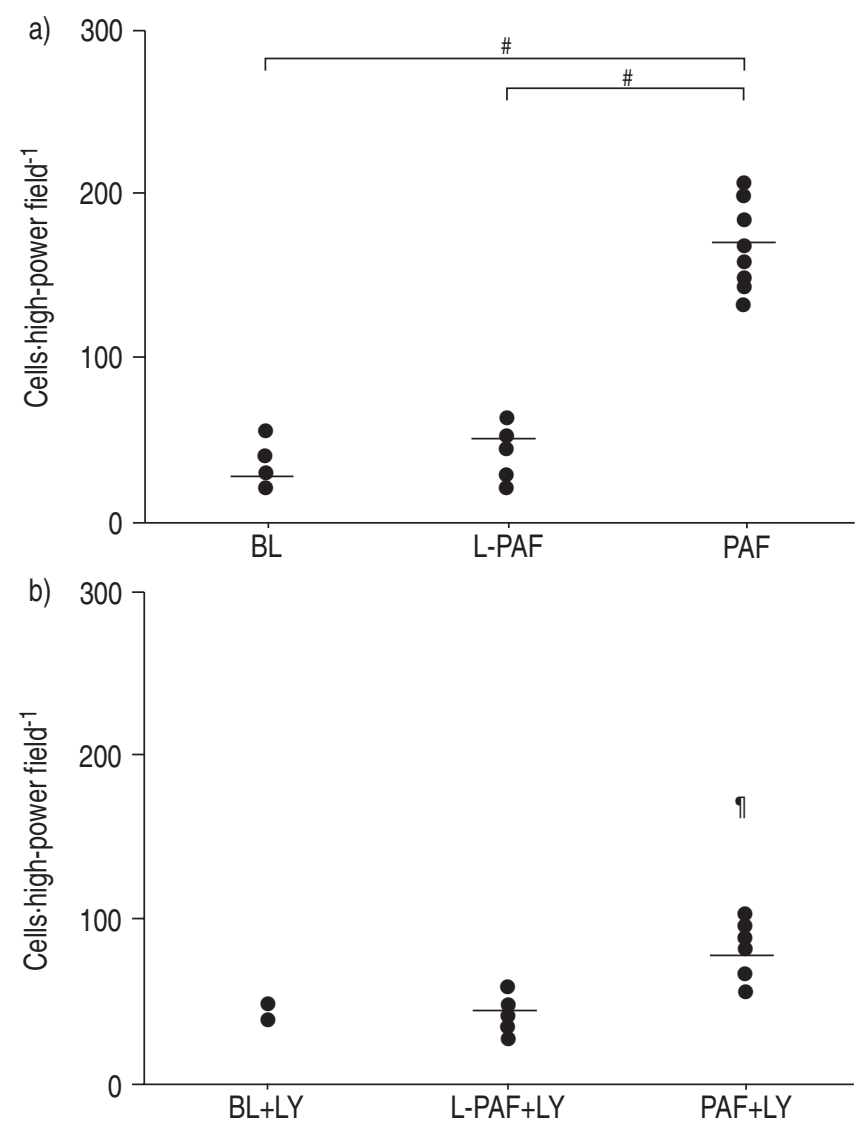

Fig. 5. - a) Chemotactic activity of induced sputum supernatant at baseline (BL) and $240 \mathrm{~min}$ after Lyso-platelet-activating factor (L-PAF) and PAF. \#: p<0.008. b) Effect of leukotriene $\mathrm{B}_{4}\left(\mathrm{LTB}_{4}\right)$ receptor antagonist (LY-223982) (LY) on induced sputum neutrophil chemotaxis activity assessed at BL (39.0 (38.7-40.7) cells $\cdot$ high-power field $^{-1}$ ) and $240 \mathrm{~min}$ after L-PAF (40.3 (38.2-43.5) cells high-power field $^{-1}$ ) and PAF. The latter was substantially inhibited compared with that induced by PAF. Data is expressed as individual migrated neutrophils per high-power field after substraction of cells migrated in the negative control (horizontal bars denote median values). 个: $\mathrm{p}<0.008$.

involved. Indeed, for leukocyte transmigration to occur within the lung parenchyma a chemotactic gradient has to be established in order to allow for the recruitment of leukocytes from the bronchial and pulmonary bloodstream into the airway and pulmonary interstitium, respectively. Among the various factors that are capable of promoting airway neutrophil chemotaxis, a central role may be played by $\mathrm{LTB}_{4}$ [12]. The results of the present study indicate that either PAF challenge released $\mathrm{LTB}_{4}$ within the airways of asthmatic patients or that $\mathrm{LTB}_{4}$ was released by neutrophils recruited and activated by PAF, or both. In addition, it was found that increased $\mathrm{LTB}_{4}$ was significantly correlated with the elevated number of sputum neutrophils recruited by the airways after PAF, thereby suggesting that $\mathrm{LTB}_{4}$ may be involved in neutrophil recruitment within the airways of patients with obstructive airway disorders [36]. This hypothesis is further supported by the in-vitro experiments that revealed the ability of sputum samples obtained after PAF to promote neutrophil chemotaxis in a significantly greater manner than that shown at baseline or after L-PAF. It was also demonstrated that the chemotactic activity of induced sputum from patients with asthma challenged with PAF is significantly greater than that exerted by sputum supernatant obtained at baseline or after L-PAF. Moreover, the data indicate an important role for sputum $\mathrm{LTB}_{4}$ in the process of neutrophil recruitment, since a
$\mathrm{LTB}_{4}$ receptor antagonist inhibited the neutrophil-chemotactic activity exerted by sputum obtained after PAF challenge. Yet, the sputum chemotactic activity of neutrophils did not correlate with its $\mathrm{LTB}_{4}$ concentrations, probably suggesting that other mediators in sputum samples, such as IL-8 and tumour necrosis factor- $\alpha$, may be involved in the process.

Asthmatic rather than normal individuals were chosen for this study for two reasons. First, it has previously been found that asthmatic patients respond to a larger degree of ventilation to perfusion imbalance than healthy individuals, leading to a greater degree of hypoxaemia in asthmatics $[15,18]$. It was assumed from this that both the inflammatory and integrin responses in asthmatics would be greater. Secondly, previous studies have indicated that neutrophils may play an important role in asthma, particularly in the more severe forms [1-5]. Accordingly, the authors wanted to confirm these observations, and if so, examine the role of some of these integrins in neutrophil accumulation, hence the current study was designed to address, at least in part, the issue of neutrophil traffic in asthma.

All in all the data point to the view that the increased leukotriene $B_{4}$ release in the airways and the subsequent enhanced recruitment of neutrophils may result from the ability of platelet-activating factor to stimulate 5-lipoxygenase activity in airway cells of asthmatic patients. This hypothesis is further supported by a previous study showing that, after platelet-activating factor exposure, neutrophils and macrophages isolated from bronchoalveolar fluid in asthmatics released a greater amount of leukotriene $B_{4}$ than in healthy individuals $[25,37]$. In summary, both $\beta_{2}$-integrins, macrophage- 1 antigen and lymphocyte function-associated antigen-1, are involved in platelet-activating factor-induced neutrophil lung sequestration in patients with mild asthma, a process that may be modulated by leukotriene $\mathrm{B}_{4}$ release within the airways. Moreover, the current laboratory-induced model of neutrophil trafficking within the lung may be of interest to further explore the mechanisms of neutrophil kinetics and their modulation by different interventions in chronic obstructive airway diseases.

Acknowledgements. The authors would like to thank M.T. Carrión, and C. Agustí for their technical advice.

\section{References}

1. Fahy JV, Kim KW, Liu J, Boushey HA. Prominent neutrophilic inflammation in sputum from subjects with asthma exacerbation. J Allergy Clin Immunol 1995; 95: 843852.

2. Lamblin C, Gosset P, Tillie-Leblond I, et al. Bronchial neutrophilia in patients with noninfectious status asthmaticus. Am J Respir Crit Care Med 1998; 157: 394402.

3. Sur S, Crotty TB, Kephart GM, et al. Sudden-onset fatal asthma. A distinct entity with few eosinophils and relatively more neutrophils in the airway submucosa? Am Rev Respir Dis 1993; 148: 713-719.

4. Jatakanon A, Uasuf C, Maziak W, Lim S, Chung KF, Barnes PJ. Neutrophilic inflammation in severe persistent asthma. Am J Respir Crit Care Med 1999; 160: 1532-1539.

5. Gibson PG, Simpson JL, Saltos N. Heterogeneity of airway inflammation in persistent asthma: evidence of neutrophilic inflammation and increased sputum interleukin-8. Chest 2001; 119: 1329-1336.

6. Wenzel SE, Szefler SJ, Leung DY, Sloan SI, Rex MD, Martin RJ. Bronchoscopic evaluation of severe asthma. 
Persistent inflammation associated with high dose glucocorticoids. Am J Respir Crit Care Med 1997; 156: 737-743.

7. Ordonez CL, Shaughnessy TE, Matthay MA, Fahy JV. Increased neutrophil numbers and IL-8 levels in airway secretions in acute severe asthma: clinical and biologic significance. Am J Respir Crit Care Med 2000; 161: 1185-1190.

8. Norzila MZ, Fakes K, Henry RL, Simpson J, Gibson PG. Interleukin-8 secretion and neutrophil recruitment accompanies induced sputum eosinophil activation in children with acute asthma. Am J Respir Crit Care Med 2000; 161: 769774.

9. in't Veen JC, Grootendorst DC, Bel EH, Smits HH, Van der Keur M, Hiemstra PS. CD11b and L-selectin expression on eosinophils and neutrophils in blood and induced sputum of patients with asthma compared with normal subjects. Clin Exp Allergy 1998; 28: 606-615.

10. Noguera A, Busquets X, Sauleda J, Villaverde JM, MacNee W, Agustí AGN. Expression of adhesion molecules and $\mathrm{G}$ proteins in circulating neutrophils in chronic obstructive pulmonary disease. Am J Respir Crit Care Med 1998; 158: 1664-1668.

11. Doerschuk CM, Tasaka S, Wang Q. CD11/CD18-dependent and -independent neutrophil emigration in the lungs: how do neutrophils know which route to take? Am J Respir Cell Mol Biol 2000; 23: 133-136.

12. Busse W. The role and contribution of leukotrienes in asthma. Ann Allergy 1998; 81: 17-26.

13. Cassatella MA, Gasperini S, Calzetti F, McDonald PP, Trinchieri G. Lipopolysaccharide-induced interleukin-8 gene expression in human granulocytes: transcriptional inhibition by interferon-gamma. Biochem J 1995; 310: 751-755.

14. Hill AD, Balley D, Stockley RA. The interrelationship of sputum inflammatory markers in patients with chronic bronchitis. Am J Respir Crit Care Med 1999; 160: 893-898.

15. Rodriguez-Roisin R, Félez MA, Chung KF, et al. Plateletactivating factor causes ventilation-perfusion mismatch in humans. J Clin Invest 1994; 93: 188-194.

16. Wardlaw AJ, Chung KF, Moqbel R, et al. Effects of inhaled PAF in humans on circulating and bronchoaveolar lavage fluid neutrophils. Relationship to bronchoconstriction and changes in airway hyperresponsiveness. Am Rev Respir Dis 1990; 141: 386-392.

17. Masclans JR, Barberà JA, MacNee W, et al. Salbutamol reduces pulmonary neutrophil sequestration by plateletactivating factor in humans. Am J Respir Crit Care Med 1996; 154: 529-532.

18. Félez MA, Roca J, Barberà JA, et al. Inhaled plateletactivating factor worsens gas exchange in mild asthma. Am J Respir Crit Care Med 1994; 150: 369-373.

19. Kilgore KS, Ward PA, Warren JS. Neutrophil adhesion to human endothelial cells is induced by the membrane attack complex: the roles of $\mathrm{P}$-selectin and platelet activating factor. Inflammation 1998; 22: 583-598.

20. Rainger GE, Rowley AF, Nash GB. Adhesion-dependent release of elastase from human neutrophils in a novel, flowbased model: specificity of different chemotactic agents. Blood 1998; 92: 4819-4827.

21. Smalley DM, Wood JG, Childs EW, Frank LL, Cheung LY. Platelet-activating factor (PAF) increases leukocyte adhesion but does not alter vessel diameter in the rat mesenteric microcirculation. Microvasc Res 1998; 56: 271-276.
22. Spoelstra FM, Berends C, Dijkhuizen B, de Monchy JG, Kauffman HF. Effect of theophylline on CD11b and L-selectin expression and density of eosinophils and neutrophils in vitro. Eur Respir J 1998; 12: 585-591.

23. Huang Q, Wu M, Meininger C, Kelly K, Yuan Y. Neutrophil-dependent augmentation of PAF-induced vasoconstriction and albumin flux in coronary arterioles. $\mathrm{Am} \mathrm{J}$ Physiol 1998; 275: H1138-H1147.

24. Gómez FP, Iglesia R, Roca J, Barberà JA, Chung KF, Rodriguez-Roisin R. The effects of 5-lipoxygenase inhibition by zileuton on platelet-activating factor-induced pulmonary abnormalities in mild asthma. Am J Respir Crit Care Med 1998; 157: 1559-1564.

25. Shindo K, Koide K, Fukumura M. Enhanced of leukotriene $\mathrm{B}_{4}$ release in stimulated asthmatic neutrophils by plateletactivating factor. Thorax 1997; 52: 1024-1029.

26. Takasaki J, Kawauchi Y, Masuho Y. Synergistic effect of type II phospholipase $\mathrm{A}_{2}$ and platelet-activating factor on MAC-1 surface expression and exocytosis of gelatinase granules in human neutrophils: evidence for the 5-lipoxygenase-dependent mechanism. J Immunol 1998; 160: 5066-5072.

27. Pizzichini E, Pizzichini MM, Efthimiadis A, et al. Indices of airway inflammation in induced sputum: reproducibility and validity of cell and fluid-phase measurements. Am J Respir Crit Care Med 1996; 154: 308-317.

28. Chung KF, Barnes PJ. Role of inflammatory mediators in asthma. Br Med Bull 1992; 48: 135-148.

29. Profita M, Sala A, Riccobono L, et al. 15(S)-HETE modulates $\mathrm{LTB}_{4}$ production and neutrophil chemotaxis in chronic bronchitis. Am J Physiol (Cell Physiol) 2000; 279: C1249-C1258.

30. in't Veen JC, de Gouw HW, Smits HH, et al. Repeatability of cellular and soluble markers of inflammation in induced sputum from patients with asthma. Eur Respir $J$ 1996; 9: 2441-2447.

31. Echazarreta AL, Dalhén B, García G, et al. Pulmonary gas exchange and sputum cellular responses to inhaled leukotriene $\mathrm{D}_{4}$ in asthma. Am J Respir Crit Care Med 2001; 164 : 202-206.

32. Walzog B, Weinmann P, Jeblonski F, Scharfftter-Kochanek K, Bommert K, Gaehtgens P. A role for $\beta_{2}$-integrins (CD11/ $\mathrm{CD} 18)$ in the regulation of cytokine gene expression of polymorphonuclear neutrophils during the inflammatory response. FASEB J 1999; 13: 1855-1865.

33. Albeida SM. Endothelial and epithelial cell adhesion molecule. Am J Respir Cell Mol Biol 1991; 4: 195-203.

34. Drost EM, MacNee W. Potential role of IL-8, plateletactivating factor and TNF- $\alpha$ in the sequestration of neutrophils in the lung: effects of neutrophil deformability, adhesion receptor expression, and chemotaxis. Eur J Immunol 2002; 32: 393-403.

35. Ding MD, Babensee JE, Simon SI, et al. Relative contribution of LFA-1 and MAC-1 to neutrophil adhesion and migration. J Immunology 1999; 163: 5029-5038.

36. Crooks SW, Stockley RA. Leukotriene $\mathrm{B}_{4}$. Int J Biochem Cell Biol 1998; 30: 173-178.

37. Shindo K, Koide K, Fukumura M. Platelet-activating factor increases leukotriene $\mathrm{B}_{4}$ release in stimulated alveolar macrophages from asthmatic patients. Eur Respir J 1998; 11: 1098-1104. 